\title{
Brain Imaging Techniques: Improving the Quality
}

\author{
Rani M. Noble, CNMT, RT(N) \\ Nuclear Medicine Department, HonorHealth, Phoenix, Arizona
}

CE credit: For CE credit, you can access the test for this article, as well as additional JNMT CE tests, online at https://www.snmmilearningcenter.org. Complete the test online no later than September 2024. Your online test will be scored immediately. You may make 3 attempts to pass the test and must answer $80 \%$ of the questions correctly to receive $1.0 \mathrm{CEH}$ (Continuing Education Hour) credit. SNMMI members will have their CEH credit added to their VOICE transcript automatically; nonmembers will be able to print out a CE certificate upon successfully completing the test. The online test is free to SNMMI members; nonmembers must pay $\$ 15.00$ by credit card when logging onto the website to take the test.

Image quality is a factor of the utmost importance in nuclear medicine. Regardless of what type of scan is being done, technologists should be diligent in how images are being acquired. With respect to brain imaging, there can be challenges that the technologist must understand how to navigate and overcome. This continuing education article, focusing mainly on brain SPECT (particularly with ${ }^{123}$ ioflupane) and brain ${ }^{18} \mathrm{~F}$-FDG PET/CT, will help technologists better understand how artifacts occur and how imaging techniques can affect image quality.

Key Words: quality; brain; techniques; artifacts

J Nucl Med Technol 2021; 49:209-214

DOI: $10.2967 /$ jnmt.120.257592

$\mathbf{T}$ here are many variables that can affect the quality of imaging. These range from technical or mechanical problems to patient and technologist issues. It is imperative that technologists obtain sufficient training to be competent in quality-imaging techniques regardless of the type of nuclear medicine scan being done. Errors in following the imaging protocol, a lack of understanding of the isotope being used, and not performing instrumentation quality control (QC) tests can affect image quality and, in turn, cause image misinterpretation and an incorrect outcome for patients.

To help technologists gain up-to-date information on the best practices for brain imaging, this article covers the proper techniques to achieve high-quality results for various types of SPECT and PET/CT brain scans. It also reviews important aspects of the various isotopes that are used.

\section{DEFINITION OF A QUALITY IMAGE}

A high-quality image produces the best possible resolution and contrast to allow for an accurate diagnosis (1). Several factors go into ensuring that a high-quality nuclear medicine image is produced. These differ according to the

Received Sep. 26, 2020; revision accepted Mar. 18, 2021.

For correspondence or reprints, contact Rani M. Noble (raninoble@ yahoo.com).

Published online July 9, 2021.

COPYRIGHT (C) 2021 by the Society of Nuclear Medicine and Molecular Imaging. imaging device being used, the patient being imaged, the isotope being used, and the technologist's capability (1). Issues that can impact the integrity of the image include suboptimal camera QC, poor spatial resolution, nonuniformity, incorrect energy resolution, inadequate patient preparation, poor technologist education or understanding of protocols, instrument artifacts, patient artifacts, inadequate patient dose, incorrect camera parameters, and mistakes in image interpretation (1). The technologist must not neglect considering all such variables. If they can be avoided, every image will be of high quality. It is especially important that protocols be standardized throughout institutions and that technologists be capable of overcoming imaging challenges when they arise.

\section{PATIENT PREPARATION}

The first step to ensuring proper imaging technique is to prepare the patient. Depending on the protocol for the particular type of scan, the patient may need to stop taking certain medications and fast for a specific time.

For ${ }^{123}$ I-ioflupane (DaTscan; GE Healthcare) SPECT, patients will need to stop taking any medication that might bind to the dopamine transporter (2), as such binding may impact the ability of ${ }^{123}$ I-ioflupane to fully bind to the dopamine transporter. Patients will also need to be prepped with an iodine solution, $400 \mathrm{mg}$ of potassium perchlorate, a single dose of potassium iodide, or a single dose of Lugol solution. Single doses are equal to $100 \mathrm{mg}$ of iodine and prevent any free ${ }^{123}$ I from being taken up by the thyroid gland (2).

Brain perfusion SPECT does not require as much patient preparation as some other scans. For $24 \mathrm{~h}$ before the scan, patients must avoid substances that can affect brain perfusion, such as alcohol, caffeine, and stimulants; cigarette smoking needs to be stopped the day of the examination (3). The technologist follows the preinjection protocol that allows for the best possible cerebral uptake. This protocol entails placing the patient in a dimly lit, quiet room; obtaining intravenous access; waiting 10-15 min for the patient to relax; making sure the patients does not read or speak for about $5 \mathrm{~min}$ before and after the injection; and injecting the radiopharmaceutical while the patient's are eyes open (closed eyes can cause 
hypometabolism of the occipital lobe and a false-positive finding of dementia with Lewy bodies) $(3,4)$.

Preinjection and postinjection preparation for brain ${ }^{18} \mathrm{~F}$ FDG PET/CT is similar to that for brain perfusion SPECT in that patients need to be in a calm, dimly lit room and intravenous access established about $10 \mathrm{~min}$ before injection of the tracer. If the injection is done any sooner, false uptake can occur in the pain center of the brain. To prevent uptake in the hearing area of the brain, the technologist should also not talk with the patient during the injection. After receiving the injection, the patient needs to keep the eyes open but avoid speaking, reading, or any considerable movement during the 30-min uptake period (5). In addition, the patient needs to fast for 4-6 h before the scan, including no alcohol, drugs, or caffeine (drinking water is acceptable), and the blood glucose level needs to be checked. The acceptable level varies by institution; most commonly, if the level is greater than $150-200 \mathrm{mg} / \mathrm{dL}$, the scan will be rescheduled for a later date when a lower level can be achieved (5).

\section{MECHANICAL ARTIFACTS}

There are several types of mechanical artifacts that can decrease image quality. It is essential that camera QC testing be performed on a regular schedule to avoid any chance of a decline in image quality. QC testing may differ among camera manufacturers; technologists need to be familiar with the routine testing required for the cameras they use.

One of the main QC procedures is daily uniformity testing. This can be either extrinsic or intrinsic, depending on the recommendation of the manufacturer, and is done mainly to make sure the detector's response to a source is within the defined limits. Uniformity testing can identify multiple different issues that degrade image quality, such as issues with the photomultiplier tubes, spatial linearity, and energy, and can reveal generalized degradation of uniformity in images ( 6 ).

Spatial-resolution, linearity, and center-of-rotation testing is also needed. Again, the specific protocol varies among manufacturers. Spatial-resolution and linearity testing help determine long-term, rather than acute, degradation of camera resolution (6) and will indicate whether something was adjusted incorrectly during maintenance. Center-of-rotation testing is important for SPECT imaging. If the center of rotation is not in a straight line across the axial direction and does not have the sine wave shape across the other axis, the center of rotation may have shifted and not be centered accurately, causing blurring and resolution degradation (6). It is important that the center of rotation be tested for each zoom and collimator.

High-count flood imaging, another QC procedure that checks for nonuniformity around the central axis of rotation, should be performed only during acceptance testing or if damage to a collimator is suspected ( 6 ). The filtered backprojection that is used during reconstruction of the flood image helps showcase any ring artifacts (6).

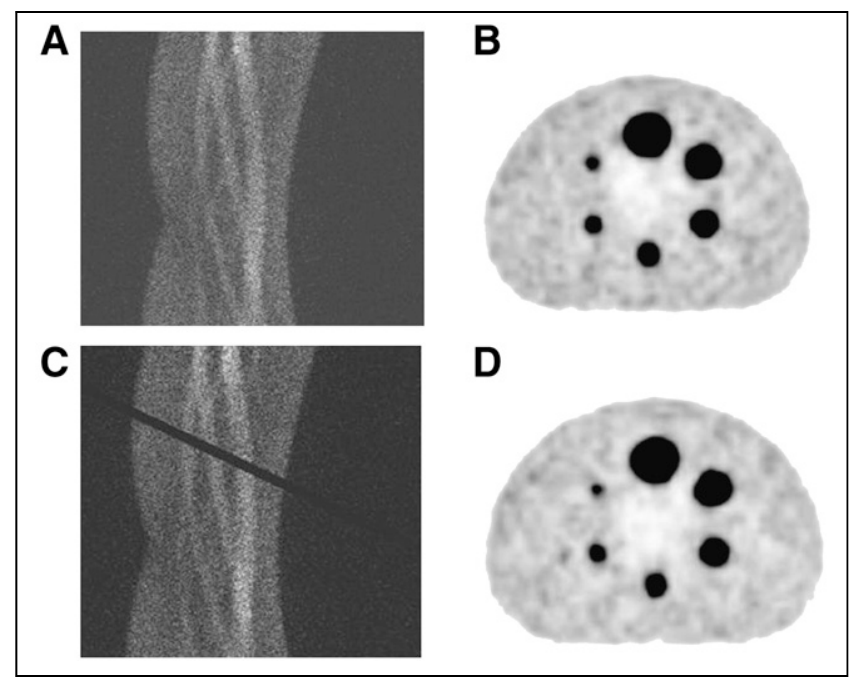

FIGURE 1. Two-dimensional PET sinograms (A and B) and corresponding reconstructed transverse PET images ( $C$ and $D$, respectively) of ${ }^{18} \mathrm{~F}$-filled phantom. Black band in $\mathrm{C}$ (suggestive of faulty detector) is virtually undetectable in $\mathrm{D}(11)$.

Neglecting to perform scheduled camera maintenance and QC tests can cause a considerable loss of image quality. The manufacturer should be notified if any of the QC results fall outside the reference limits it sets. If a camera with undesirable QC values continues to be used, patient images can show inaccurate findings and degraded quality and resolution, potentially affecting patient care and treatment (Fig. 1).

\section{TECHNOLOGIST-TRIGGERED ARTIFACTS}

Technologists need to have adequate training in the nuclear medicine procedures they perform. A lack of understanding about the procedure can lead to technologist-triggered artifacts that can, in turn, result in uninterpretable images. Some such errors include poor image processing, lack of patient education or preparation, neglecting to monitor for patient motion, use of the incorrect dose, and improper operation of the camera (Fig. 2).

Avoiding these errors is especially important in brain imaging. A mistake such as not positioning the detectors at the correct distance from the patient can impact image quality. For example, the radius of the detectors relative to the patient for a ${ }^{123}$ I-ioflupane scan should be between 11 and $15 \mathrm{~cm}$ (2). A larger radius can cause blurring (Fig. 3). The technologist needs to be familiar with how many counts are adequate for the scan being done, as well as the correct scan length. ${ }^{123}$ I-ioflupane scans require a minimum of 1.5 million counts for the entire scan (2). The scan time for PET/CT depends on the type of camera. Brain SPECT requires 60 or more stops, and the scan length should be limited to $30-45 \min (7)$ to reduce patient discomfort and motion. Insufficient counts or camera stops can produce suboptimal images that may be uninterpretable.

Constant monitoring of the imaging facility by the technologist and management can reveal practices and performance 


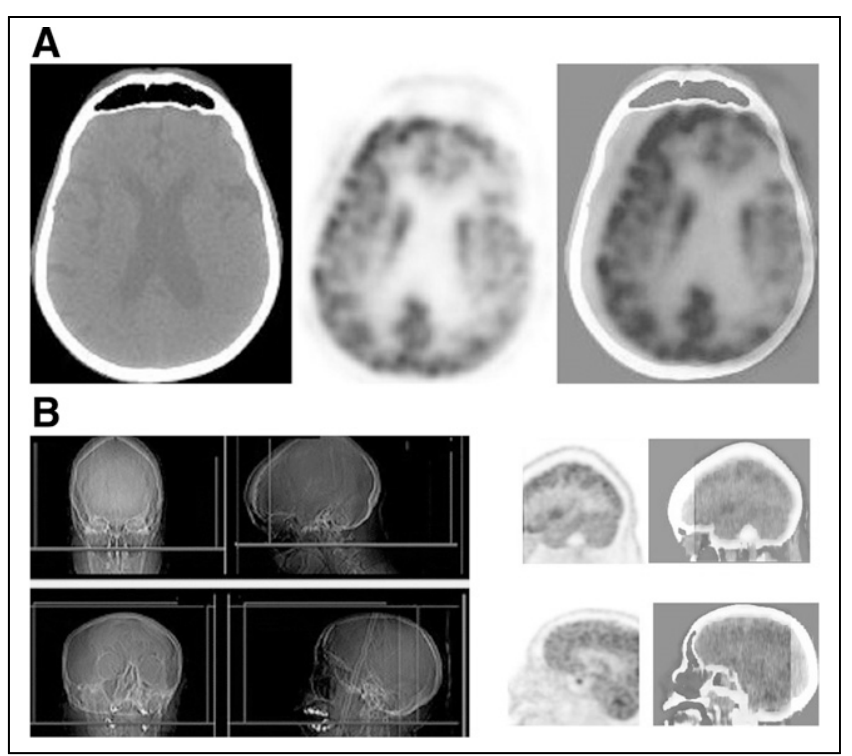

FIGURE 2. (A) Brain ${ }^{18} \mathrm{~F}-\mathrm{FDG}$ PET/CT images with technologistcaused motion artifact due to misplacing of region-of-interest box on scout images. (B) Transaxial and sagittal lateral radiographs (left), PET images (middle), and PET/CT images (right) of head. Artifact causes false reduction in uptake in left hemicortex and results in part of brain being cut off in final images (12).

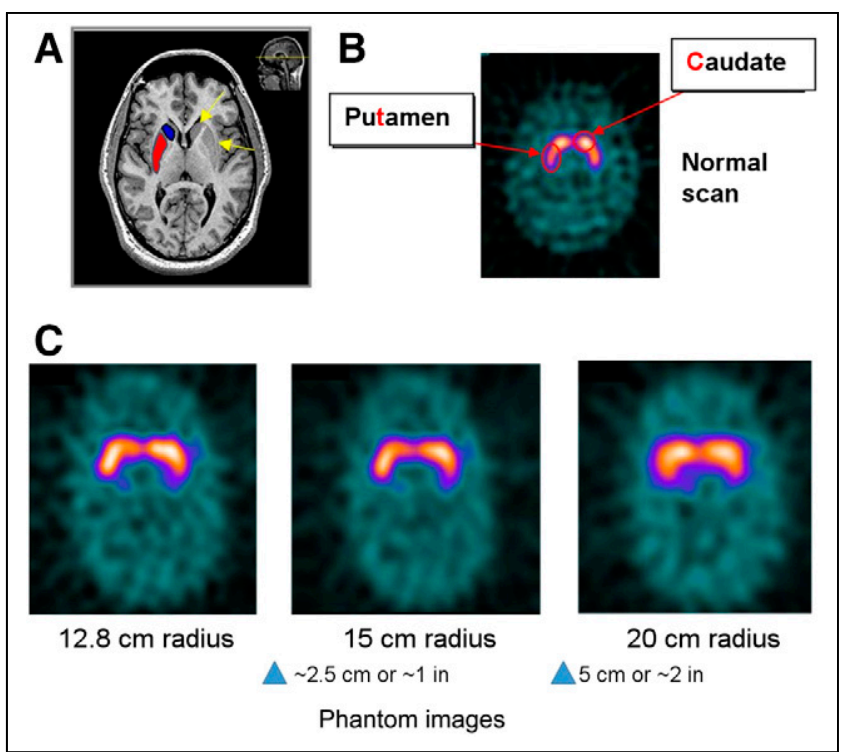

FIGURE 3. (A and B) Brain MRI (A) and PET (B) scans showing location of caudate nucleus and putamen within brain. These are main focus of ${ }^{123}$ I-ioflupane scans and need to be fully included in images. (C) PET phantom scans showing how important it is to keep detector radius within $11-$ to $15-\mathrm{cm}$ range. More distance between patient and detector will increase blurring (9). Arrows in A point to left caudate and left putamen; blue indicates right caudate and red indicates location of right putamen. Scans in A and $\mathrm{B}$ are of a patient.

that need improvement. One option for improving quality within the imaging facility is to use data analysis to examine performance. A previously published practice guideline laid out a useful 10-step process to help facilities improve the quality of performance within their lab (8). The 10 steps of quality improvement include identifying a potential problem or area of concern, gathering information and understanding the full extent of the problem, stating the goal and setting goal targets, designing a data collection strategy, collecting data, analyzing those data, sharing the results of the data, implementing the needed changes, recollecting data, and analyzing. Following these 10 steps can identify suboptimal performance and provide an opportunity for improvement. A step-by-step checklist plan such as this one- that helps reduce technologist-triggered artifacts and imaging mistakes - can reduce the number of patients who need to be rescanned.

\section{PATIENT POSITIONING}

Patient positioning is one of the more critical steps in a protocol. A head holder should be used for brain imaging. Before getting onto the imaging table, patients should be encouraged to void to prevent interruption of the scan. Patients also need to remove eyeglasses, hats, earrings, and any other items that can interfere with the images. The technologist should help the patient get as comfortable as possible on the table, such as by providing a knee bolster to alleviate pressure on the lower back or a blanket for warmth. A body strap can be used to secure the patient in a comfortable position on the table, and straps across the forehead or chin can also be used to help prevent motion during imaging $(9)$.

The canthomeatal line is a standard positioning line used for most brain scans. It is an imaginary line extending from the lateral canthus to the center of the auditory meatus and should be perpendicular to the imaging table. Basing the patient positioning on this imaginary line will help ensure that the entire brain is included in the imaging field. However, it is more important that the patient be in a comfortable position than for this line to be perpendicular to the imaging table. In such a case, proper orientation of the patient's brain can be achieved during processing, and the interpreting physician should be told that the patient was difficult to position (Fig. 4).

\section{PRECAUTIONS}

Technologists need to take into consideration the type of patient and the type of brain scan and remain flexible about

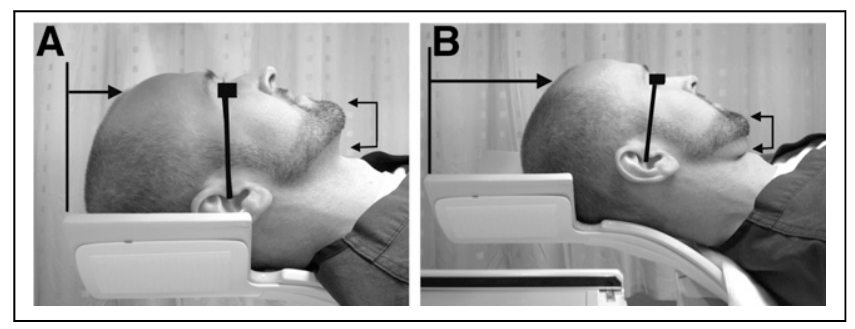

FIGURE 4. (A) Correct positioning of head during brain imaging. Canthomeatal line should be positioned as perpendicularly to imaging table as possible to eliminate head tilt and resulting artifacts. (B) Incorrect head positioning. Patient's head is tilted and not placed fully in head holder (13). 
applying extra care and precautions if needed. For example, because patients might move during ${ }^{123}$ I-ioflupane imaging, securing and immobilizing the head are extremely important. Extra time should be taken to ensure proper head placement, and chin and head straps should be used to ensure that the patient remains still during the entire scan. Another example is a brain ${ }^{18} \mathrm{~F}$-FDG PET/CT scan being performed on a patient who might be cognitively impaired and not able to remember instructions about remaining still on the imaging table. These instructions may need to be repeated several times during the scan.

Another important precaution technologists can take is to monitor and give special attention to patients with neurologic defects (10), who might find it difficult to complete a brain scan. Monitoring will ensure that the patient is comfortable and still throughout the entire scan. Straps across the forehead and chin may help keep the patient's head correctly positioned and motion-free during the scan. Monitoring is especially important for patients with epilepsy and can include continuous recording if the patient is being scanned as part of a preoperative evaluation.

It is not uncommon for patients to need a mind-altering drug, such as a sedative, to help them tolerate a scan. If sedation is needed for a brain PET/CT scan, it should be given as late as possible after the uptake period (5). If needed for a
${ }^{123}$ I-ioflupane scan (and depending on the sedation), it should be given anywhere from $30 \mathrm{~min}$ to immediately before imaging to provide ample time for the sedation to take effect (2). If needed for a brain SPECT scan, it should be given 5 min after injection of the radiopharmaceutical (10). Sedation can be helpful for patients who are claustrophobic, have difficulty lying still, have dementia, or are uncooperative.

\section{IMAGE INTERPRETATION}

Image quality should be checked before image interpretation. Cine or sinogram images should be checked for motion and head alignment. Target-to-background ratio and other artifacts such as contamination, attenuation, patient motion, and undesired radiopharmaceutical uptake should be assessed. If there are difficulties with patient motion, images can be acquired in list mode and then reconstructed into a shorter-interval acquisition in the case of movement at the last minute. Precaution needs to be taken when levels of contrast are selected, as some color scales can be misleading during interpretation of results (5). Another consideration for interpretation criteria is the extent of normal variability between patients. Radiologists should familiarize themselves with a reference database (2). The general distribution of the radiotracer and symmetry between hemispheres should be observed.

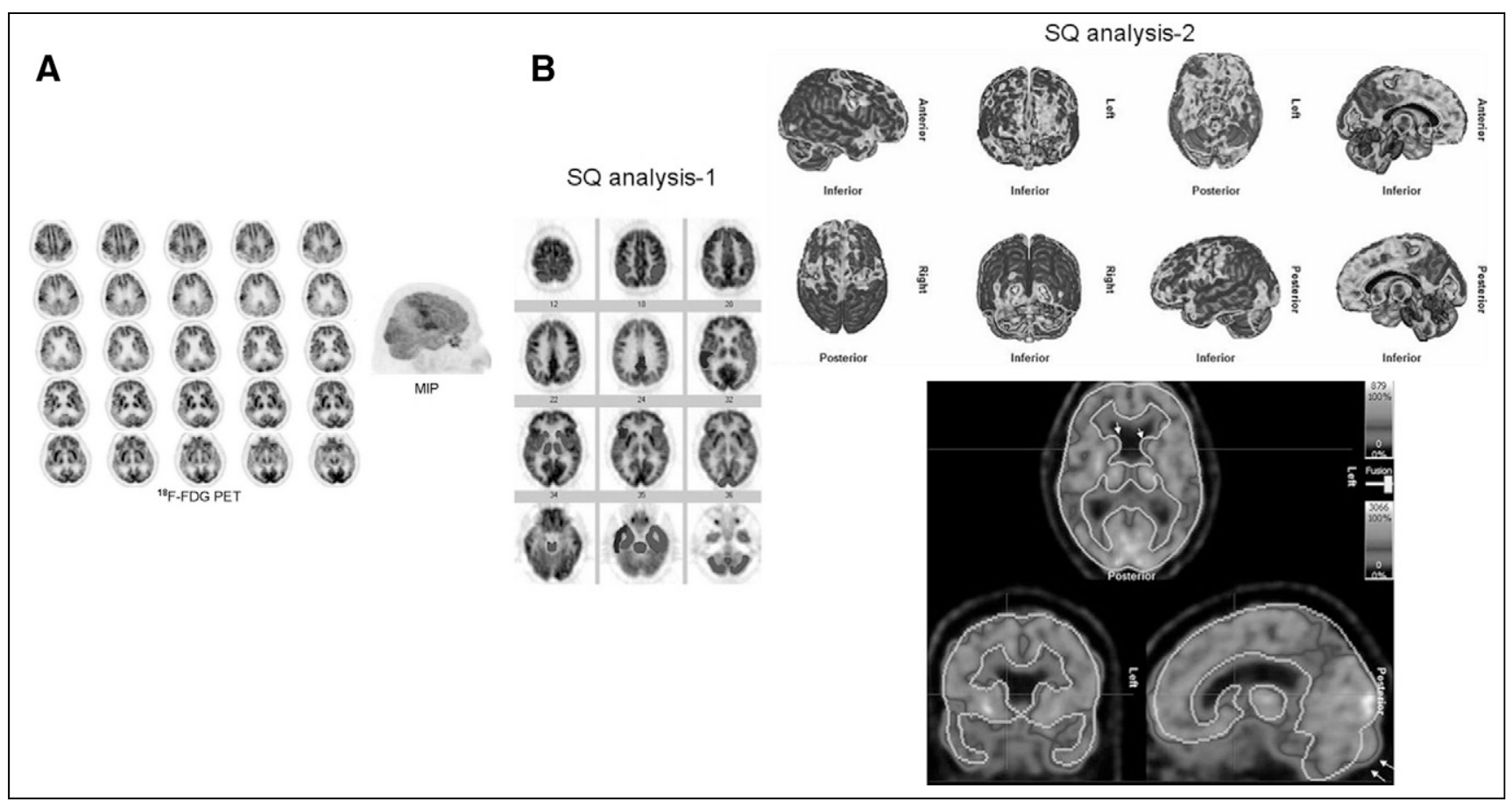

FIGURE 5. ${ }^{18}$ F-FDG PET brain images showing bilateral hypometabolism in temporal, parietal, and frontal lobes, and maximum-intensity projection (MIP) showing cerebral cortical hypometabolism localization. (B) Semiquantitative (SQ) analysis showing reduction in metabolism in patient's frontal and temporal lobes and in posterior cingulate cortices and temporoparietal junctions. Semiquantitative analysis, however, does not show hypometabolism in patient's parietal lobes and undervalues reduction of metabolism in left temporal lobe (12). In B, SQ1 uses NeuroQ software; it presents reduced metabolism in bilateral frontal lobes, posterior cingulate cortices, temporoparietal junctions, bilateral temporal lobes with right being greater than left, and mildly in left basal ganglia. SQ2 uses Hermes BRASS software. This software furnishes greater matching results to visual analysis in cerebral cortex. This does not apply to basal ganglia, however. This program exhibits inferior registration of various parts of brain, which includes caudate heads. 


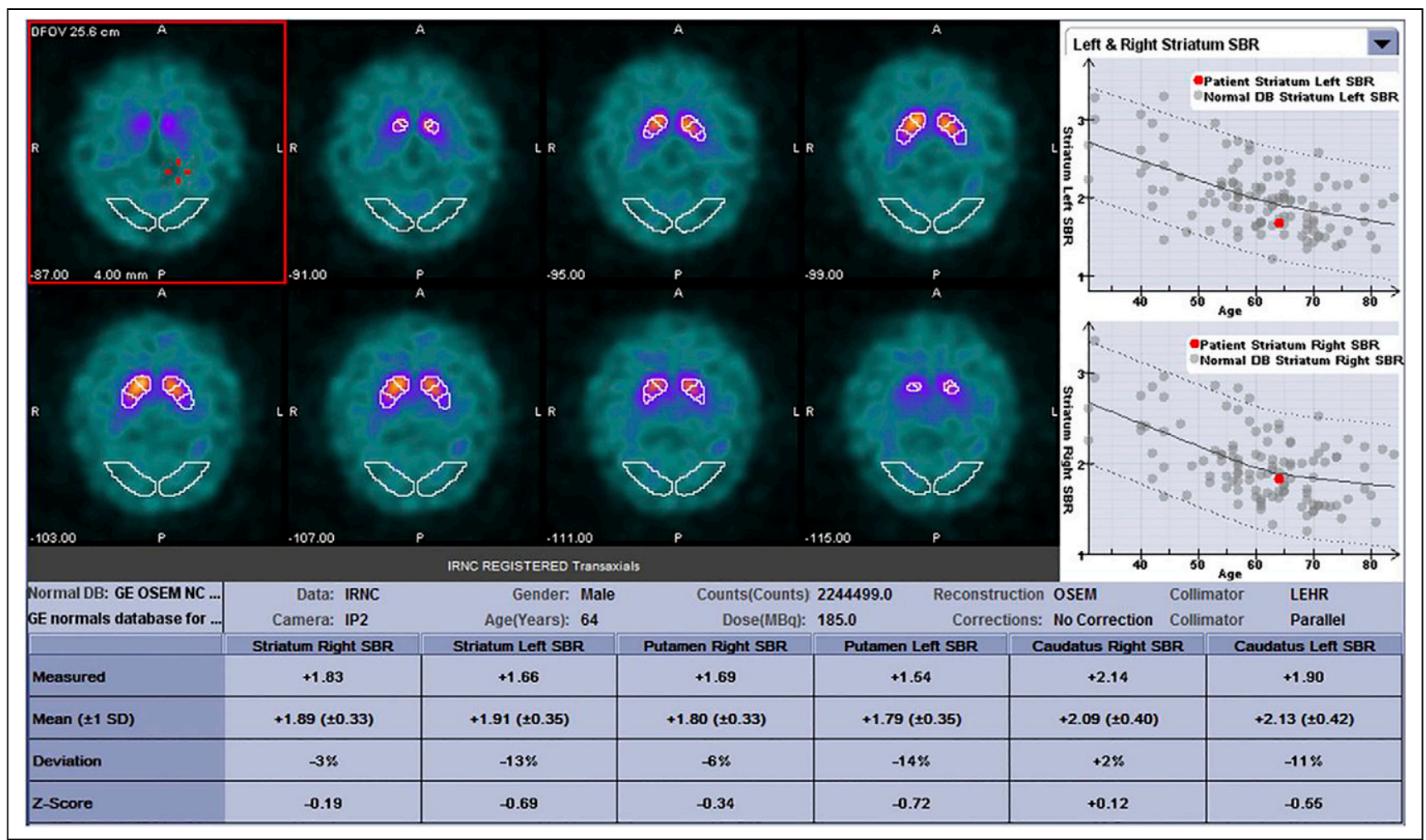

FIGURE 6. Screenshot showing results of DaTQUANT, semiquantitative analysis software used for ${ }^{123}$ I-ioflupane scans. Scans show how results would be displayed with $\mathrm{ROIs}$ placed (14). $\mathrm{A}=$ anterior; $\mathrm{DB}=$ database; IP2 = type of operating system in Siemens cameras, computer software; IRNC = iterative reconstruction without attenuation correction; LEHR = low-energy high-resolution; NC = normal control; OFOV = operational field of view; OSEM = ordered-subsets expectation maximization; $\mathrm{P}=$ posterior; $\mathrm{SBR}=$ striatal binding ratio.

Qualitative or semiquantitative analysis can be done for brain image interpretation. Brain PET/CT scans will differ from routine oncologic PET/CT scans because an SUV is not used; however, quantitative analysis can still be done. Absolute glucose metabolism can be measured to calculate the arterial ${ }^{18} \mathrm{~F}$ FDG concentration-against-time curve. This calculation would require serial sampling of arterial blood (Fig. 5) (5).

Semiquantitative analysis can complement visual interpretation for ${ }^{123}$ I-ioflupane scans. To be interpretable, the semiquantitative data need to be compared with a reference database (2). However, the reference database will need further clarification regarding what makes it "normal." Not using an automated system for regions of interest can cause interobserver variability, resulting in region-of-interest placement errors and false interpretations (2). Automated ROI placement systems help reduce that variability. Several different studies have shown accurate diagnoses from interpretations based on only semiquantitative information. However, these results were only for experienced readers (2). There is no information on whether inexperienced readers would have the same outcome, but semiquantitative information may benefit those readers with less experience (Fig. 6) (2).

\section{CONCLUSION}

Quality imaging starts at the technologist level. Understanding brain imaging and how to accomplish high-quality images is very achievable. Brain imaging is a critical part of the nuclear medicine field, and high-quality images can help in the diagnosis of crucial conditions. Every step of the protocol should be followed, from patient preparation, education, and positioning to camera operation and image interpretation. Images that are submitted for interpretation should be of the best quality obtainable. Achieving this level of quality may mean having to overcome challenges that arise during the imaging process. If care is taken to understand a protocol completely, these challenges can be overcome and highquality images obtained.

\section{ACKNOWLEDGMENT}

I thank Mary Beth Farrell for editorial assistance, mentorship, and continued support.

\section{REFERENCES}

1. Image quality and quality control in diagnostic nuclear medicine. International Atomic Energy Agency website. https://www.iaea.org/resources/rpop/health-professionals/ nuclear-medicine/diagnostic-nuclear-medicine/image-quality-and-quality-control. Accessed June 28, 1958.

2. Djang DSW, Janssen MJR, Bohnen N, et al. SNM practice guideline for dopamine transporter imaging with ${ }^{123}$ I-ioflupane SPECT 1.0. J Nucl Med. 2012;53:154-163. 3. Kapucu OL, Nobili F, Varrone A, et al. EANM procedure guideline for brain perfusion SPECT using ${ }^{99 \mathrm{~m}} \mathrm{Tc}$-labelled radiopharmaceuticals, version 2. Eur J Nucl Med Mol Imaging. 2009;36:2093-2102.

4. Brown RK, Bohnen N, Wong K, et al. Brain PET in suspected dementia: patterns of altered FDG metabolism. Radiographics. 2014;34:684-701. 
5. Waxman AD, Herholz K, Lewis D. Society of nuclear medicine procedure guideline for FDG PET brain imaging. Society of Nuclear Medicine and Molecular Imaging website. http://s3.amazonaws.com/rdcms-snmmi/files/production/public/docs/Society $\% 20$ of $\% 20$ Nuclear $\% 20$ Medicine $\% 2$ Procedure $\% 20$ Guideline $\% 20$ for $\% 20$ FDG $\% 20$ PET\%20Brain\%20Imaging.pdf. Published February 8, 2009. Accessed June 28, 2021.

6. Murphy PH. Acceptance testing and quality control of gamma cameras, including SPECT. J Nucl Med. 1987;28:1221-1227.

7. Parker JA, Graham LS, Royal HD, et al. The SNM procedure guideline for general imaging 6.0. Society of Nuclear Medicine and Molecular Imaging website. http:// s3.amazonaws.com/rdcms-snmmi/files/production/public/docs/General_Imaging_

Version_6.0.pdf. Published January 14, 1996. Revised September 12, 2010. Accessed June 28, 2021.

8. Farrell MB, Abreu SH. A practical guide to quality improvement in nuclear medicine. J Nucl Med. 2012;40:211-219.
9. Grabher BJ. Brain imaging quality assurance: how to acquire the best brain images possible. J Nucl Med Technol. 2019;47:13-20.

10. Juni JE, Waxman AD, Devous MD Sr, et al.; Society for Nuclear Medicine. Procedure guideline for brain perfusion SPECT using $99 \mathrm{mTc}$ radiopharmaceuticals 3.0. J Nucl Med Technol. 2009;37:191-195.

11. Zanzonico P. Routine quality control of clinical nuclear medicine instrumentation: a brief review. J Nucl Med. 2008;49:1114-1131.

12. Sarikaya I, Sarikaya A, Elgazzar A. Current status of ${ }^{18}$ F-FDG PET brain imaging in patients with dementia. J Nucl Med Technol. 2018;46:362-367.

13. Covington MF, McMillan NA, Avery RJ, Kuo PH. The semicolon sign: dopamine transporter imaging artifact from head tilt. J Nucl Med Technol. 2013;41: 105-107.

14. Booij J, Dubroff J, Pryma D, et al. Diagnostic performance of the visual reading of 123I-ioflupane SPECT images with or without quantification in patients with movement disorders or dementia. J Nucl Med. 2017;58:1821-1826. 